\title{
Preparation and Characterization of Electrically and Thermally Conductive Polymeric Nanocomposites
}

\author{
Mousam Choudhury, Smita Mohanty, Sanjay K. Nayak", Rakesh Aphale \\ Laboratory for Advanced Research in Polymeric Materials (LARPM), Central Institute of Plastics \\ Engineering \& Technology, Bhubaneswar, India \\ Email: *drsknayak@yahoo.com
}

Received June 1, 2012; revised July 5, 2012; accepted July 22, 2012

\begin{abstract}
The dielectric properties of composites and nanocomposites composed of epoxy resin as base matrix and AlN (Aluminum Nitride) as micro and nanofiller has been studied at variable loading of AlN. To improve the dispersion of the filler within the polymer matrix, AlN was surface modified with silane coupling agent (SCA). The thermal conductivity behavior of epoxy/AIN composites and nanocomposites has been studied at variable percentage of filler and temperatures. Test result indicated an increase of thermal conductivity of the composites at $20 \mathrm{wt} \%$ of AlN. Also, silane treated composites exhibited improved electrical conductivity properties wherein the electrical insulation property decreased in terms of di-electric strength and resistivity.
\end{abstract}

Keywords: Epoxy; Micro-AlN; Nano-AlN; Insulation; Electrical Properties; Resistivity

\section{Introduction}

Ceramic filler reinforced polymer composites have generated considerable interest in the recent years in the areas as of electronic packaging. Relative case of processibility and excellent flexibility in these composites has driven them as potential candidates for development of devices for electric stress control and high storage capability and high permittivity material.

Epoxy, a versatile thermoset resin has been widely utilized as a packaging and insulating material in electronic and electrical industries, due to its high resistivity, low dielectric constant and excellent processibility. However, certain impediments like low thermal conductivity and high CTE of these polymers have resulted in thermal failure during its enduse application. Moreover, in addition to thermal and electrical properties, mechanical properties of epoxy resin as a substrate and packaging material also plays a vital role.

Several investigation pertaining to improving the stiffness and strength in the electronic packaging materials with the use of inorganic fillers like $\left(\mathrm{Al}_{2} \mathrm{O}_{3}, \mathrm{SiO}_{2}\right.$, $\mathrm{ZnO}$ and $\mathrm{BeO}$ ) [1-6] have been suggested. Similar other fillers such as silicon carbide $\mathrm{SiC}[3,7,8]$, nitride (AlN and $\mathrm{BN})$ [1-3,5,7-18] and carbon based materials are also known to effectively resolve the problems of thermal dissipation.

Aluminium nitride (AlN), an inorganic/ceramic filler

"Corresponding author. with high intrinsic thermal conductivity $(319 \mathrm{~W} / \mathrm{mK})$ [19] high electrical resistivity $\left(>10^{14} \Omega \cdot \mathrm{cm}\right)[20]$, low dielectric constant (8.9 at $1 \mathrm{MHz}$ ) [20] and low cost has generated considerable research interest as a reinforcing agent in the recent years. Various researches on polymer/AlN composites with modified CTE, tensile strength and dielectric constant have been performed and reported.

In the present study, AlN-epoxy composites prepared using solvent casting technique has been investigated. AIN particles at micro and nano levels have been used as a reinforcing filler to evaluate its effect on thermal and electrical conductive properties of the epoxy resin. Also AlN has been modified using wet-reflux method and silane based coupling agent has been used to improve the compatibility with the matrix polymer. Various properties such as dielectric strength, volume and surface resistivity, thermal conductivity of the composites and nanocomposites have been studied. Also, the morphology of the composites has been investigated employing scanning electron microscopic technique.

\section{Experimental}

\subsection{Raw Materials}

Aluminium Nitride (AIN) with an average diameter of less than $4 \mu \mathrm{m}$ was obtained from M/s Accer chemicals, Mumbai. Nano size AlN of less than $100 \mathrm{~nm}$ was procured from Aldrich chemicals.

The epoxy resin used in the present study was liquid 
diglycidyl ether of Bisphenol-A type (Araldite LY556) with an equivalent weight per epoxide group of 195 $\mathrm{g} / \mathrm{mol}$, has been supplied by M/s Marshal polymers, Kolkata, India. The hardener Triethylene tetramine (TETA, HY951) was also obtained from M/s Marshal Polymers, Kolkata, India.

$\gamma$-Aminopropyl-triethoxysilane (A1100) with a chemical formula $\mathrm{C}_{6} \mathrm{H}_{17} \mathrm{NO}_{3} \mathrm{Si}$, having a density $1.027 \mathrm{~g} / \mathrm{cc}$ was purchased and used as a coupling agent, from M/s Sigma Aldrich, India. All other reagents of AR grade have been used as such without any further modification.

\subsection{Surface Modification of AIN Particles}

The surface modification of AIN Particles using $\gamma$-aminopropyl-triethoxysilane coupling agent was carried out using wet-reflux method. In wet reflux method the ethanol/water/AlN mixture was sonicated for 90 minutes with addition of $10 \%$ silane by weight of AlN. The amount of coupling agent used, was $10 \mathrm{wt} \%$ based on the weight of the AlN powder.

Coupling agent/ethanol solution was slowly added to $90 \%$ ethanol and 10\% water mixture by a dropping funnel. Then the mixture was stirred continuously for $2 \mathrm{~h}$ and AlN powder was added subsequently. The coupling agent/ethanol/AIN powder mixture was stirred using an electric mixer for $3 \mathrm{~h}$ at $80^{\circ} \mathrm{C}$. The resulting slurry was centrifuged at $12000 \mathrm{rpm}$ for $15 \mathrm{~min}$ and then washed with ethanol. The surface modification of particles using $\gamma$-aminopropyl-triethoxysilane is represented in the reaction scheme (Figure 1).

\subsection{Synthesis of Epoxy/AIN Micro and Nanocomposites Using Casting Technique}

Epoxy/AlN micro and nanocomposites were prepared using casting technique, initially; required quantity of AIN powder was added to the epoxy resin and stirred. Then the hardener TETA of desired amount was added to the epoxy/AIN mixture and resulting mixture was stirred vigorously to ensure homogenous dispersion of the AIN within the epoxy matrix. Finally, the mixture was poured onto stainless steel mold, pre-cured in an oven at $135^{\circ} \mathrm{C}$ for $3 \mathrm{~h}$ and then maintained for $12 \mathrm{~h}$ at ambient temperature. The post curing was carried out at $80^{\circ} \mathrm{C}$ for $2 \mathrm{~h}$, $100^{\circ} \mathrm{C}$ for $1 \mathrm{~h}$ and $120^{\circ} \mathrm{C}$ for $2 \mathrm{~h}$ respectively. Then the mold were left in the oven and allowed to cool gradually to room temperature. Similar procedure was also employed for preparation of epoxy/AIN nanocomposite. In case of epoxy/AIN microcomposites both surface-treated and untreated AlN have been fabricated. The compositions in both surface-treated and untreated epoxy/AlN composites are maintained the same as shown in the Table 1. Various compositions prepared have been highlighted in the Table 1.

\subsection{Characterization}

\subsubsection{Density Test of Composites and Nanocomposites}

The density test of the epoxy/AIN composites and nanocomposites was measured by the Archimedes principle using alcohol as the medium. The theoretical densities in the sample were calculated based on the density of AIN of $3.26 \mathrm{~g} / \mathrm{cm}^{3}$ and the measured density of the epoxy. The volume percent of filler was determined from the density of the neat epoxy and filler.

\subsubsection{Dielectric Breakdown Strength}

Dielectric breakdown strength was measured using OTS$100 \mathrm{AF} / 2$, alternating current dielectric strength tester (Meggera OTS, UK) in accordance with ASTM D 149 2004. Specimen of $50 \mathrm{~mm}$ dia and $3 \mathrm{~mm}$ thickness were placed between two $10 \mathrm{~mm}$ diameter copper ball electrodes and the electrode system containing the measured sample was immersed in the pure silicone oil to prevent the surface flashover. The test voltage was applied across two ball-typed electrodes and was increased until the sample was punctured. Five breakdown tests were repeatedly performed on each specimen. All of the measurements were performed under the same humidity and temperature.

\subsubsection{Volume Resistivity}

Volume resistivity in the samples was measured using Ultra Megaohm Meter (SM-8220, TOA Electronics Ltd., Japan) in accordance with ASTM D 257. Square samples of $100 \mathrm{~mm} \times 100 \mathrm{~mm} \times 8 \mathrm{~mm}$ were taken for measurement of the resistivity in the samples.

\subsubsection{Thermal Conductivity}

The thermal conductivity $(\mathrm{W} / \mathrm{mK})$ was calculated by the product of the thermal diffusivity $\left(\mathrm{mm}^{2} / \mathrm{s}\right)$, specific heat $(\mathrm{J} / \mathrm{gK})$ and density $\left(\mathrm{g} / \mathrm{cm}^{3}\right)$, using Unitherm 2022 (Anter Corpo, USA) thermal conductivity tester according to

Table 1. Composition of epoxy/AIN micro and nanocomposite.

\begin{tabular}{cccc}
\hline Specimen Composition & $\begin{array}{c}\text { Epoxy } \\
\mathbf{( \% )}\end{array}$ & $\begin{array}{c}\text { Hardener } \\
(\mathbf{\%})\end{array}$ & $\begin{array}{c}\text { AIN } \\
(\%)\end{array}$ \\
\hline Epoxy + Hardener + 0\% AlN & 88 & 12 & 0 \\
Epoxy + Hardener + 5\% AlN & 83 & 12 & 5 \\
Epoxy + Hardener $+10 \%$ AlN & 78 & 12 & 10 \\
Epoxy + Hardener $+20 \%$ AlN & 68 & 12 & 20 \\
Epoxy + Hardener $+30 \%$ AlN & 58 & 12 & 30 \\
Epoxy + Hardener $+5 \%$ nAlN & 83 & 12 & 5 \\
Epoxy + Hardener $+20 \%$ nAlN & 68 & 12 & 20 \\
\hline
\end{tabular}




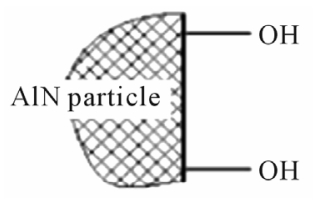<smiles>CO[Si](CCCOC1CO1)(OC)OC</smiles>

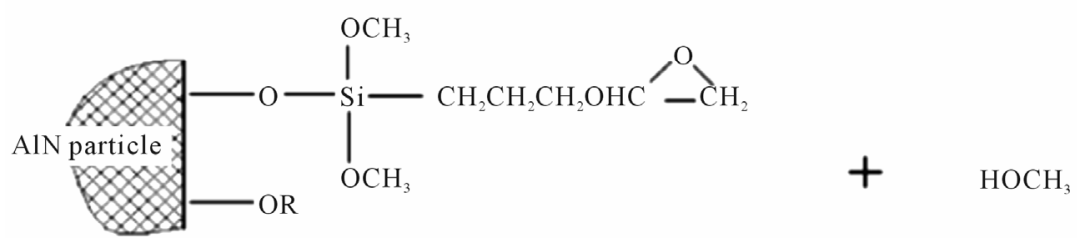

Figure 1. The schematic reactions of AIN particles and silane.

ASTM E 1530. Circular samples of $50 \mathrm{~mm}$ diameter and $3 \mathrm{~mm}$ thickness, flat on both the sides were taken for measurement of thermal conductivity. The samples were coated with a thin graphite layer on both front and back sides to increase the emission/absorption behavior at variable temperature conditions at $25^{\circ} \mathrm{C}, 50^{\circ} \mathrm{C}$ and $75^{\circ} \mathrm{C}$ respectively.

\subsubsection{Thermo Gravimetric Analysis (TGA)}

The thermal stability in surface-treated and untreated AlN particles along with the composite and nanocomposite samples have been studied employing TGA analysis (TGA, Q50, TA Instruments, USA). All the samples were scanned from $50^{\circ} \mathrm{C}$ to $600^{\circ} \mathrm{C}$ at a rate of $10^{\circ} \mathrm{C} / \mathrm{min}$ under $\mathrm{N}_{2}$ atmosphere. Corresponding degradation temperatures and percentage char have been reported.

\subsubsection{Dynamic Mechanical Analysis (DMA)}

DMA was carried out for composites and nanocomposites using a dynamic mechanical thermal analyzer ( $Q$ $800, \mathrm{M} / \mathrm{s}$ TA Instruments, USA). The samples were scanned in tension mode from $30^{\circ} \mathrm{C}$ to $200^{\circ} \mathrm{C}$ at a heating rate of $10^{\circ} \mathrm{C} / \mathrm{min}$ and fixed frequency of $1 \mathrm{~Hz}$. Corresponding dynamic modulus, loss factor and $\mathrm{Tg}$ was determined.

\subsubsection{Scanning Electron Microscopy (SEM)}

The dispersion characteristics of the epoxy/AlN composites and nanocomposites have been studied employing scanning electron microscope (EVMA-15 Carl Zeiss, UK). Prior to imaging a thin section of the fractured surface of the sample was mounted on the aluminium stub using a conductive silver paint and was coated with gold prior to fractographic examination.

\section{Results and Discussion}

\subsection{Modification of AIN Particles}

The grafting percentage of the silane coupling agent was determined by TGA measurements. For exact result, the surface treated AIN nanoparticles were washed several times to ensure complete removal of free silane coupling agent or traces of any physically bonded silane molecules.

As evident from Figure 2, the TGA thermogram of untreated and treated AIN microparticles, the weight loss in case of treated AlN particles were higher than that of the untreated particles over the entire experimental range at lower temperature region $<200^{\circ} \mathrm{C}$, the weight loss in both the Unsurface-treated and treated AIN particles is probably due to the removal of water molecules absorbed on surface of AlN.

The significant weight loss in the surface-treated AIN particles in the temperature range of $75^{\circ} \mathrm{C}-650^{\circ} \mathrm{C}$, is probably related to the condensation of silanol groups and the decomposition of grafted silane molecules [21]. This further confirms that the silane groups are successfully bonded onto the surface of the AlN particle.

\subsection{Density of Composites}

The interaction of the filler with the polymer matrix is a complex phenomenon that is usually influenced by factors that includes free volume; molecular weight; which in turn is influenced by its density. Figure 3 shows the epoxy/surface-treated AIN composite showing higher density as compared with epoxy/unsurface-treated AIN composite which may be attributed towards the presence of more volume fraction of voids and pores in epoxy/ unsurface-treated AIN composites.

The crosslink density of the composite sample is sensitive towards its volume fraction of voids and pores. As the grafting silanes on the surface of AlN particles enhanced the interaction between the matrix and the filler (AIN); it further reduces the free spaces among the epoxy molecules and increases the congestion or difficulty of epoxy molecules to either rotate or move. This further contributes to an increase in the density of the composites. 


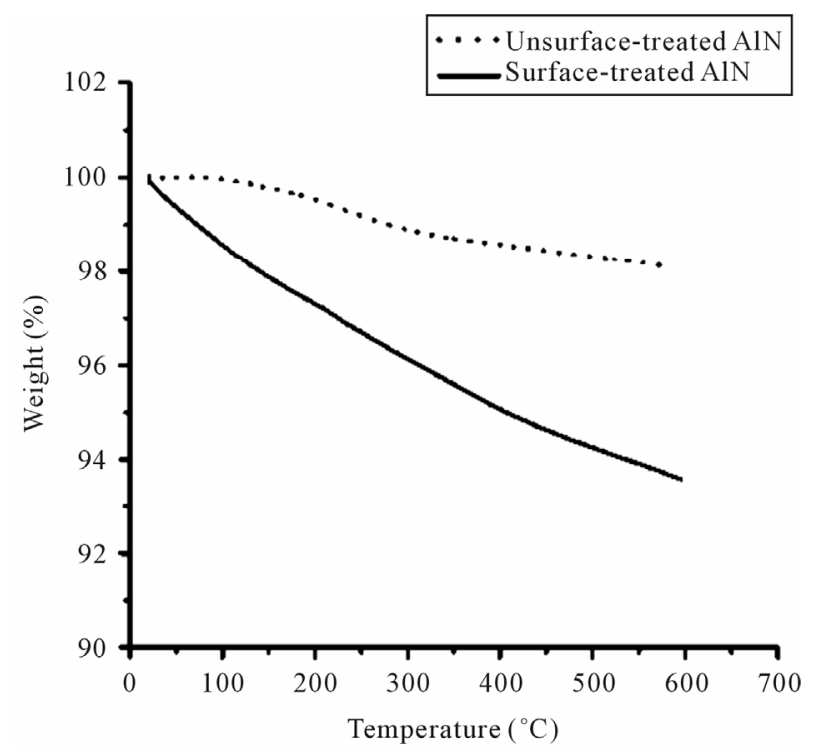

Figure 2. TGA Thermogram of untreated \& treated AIN particles.

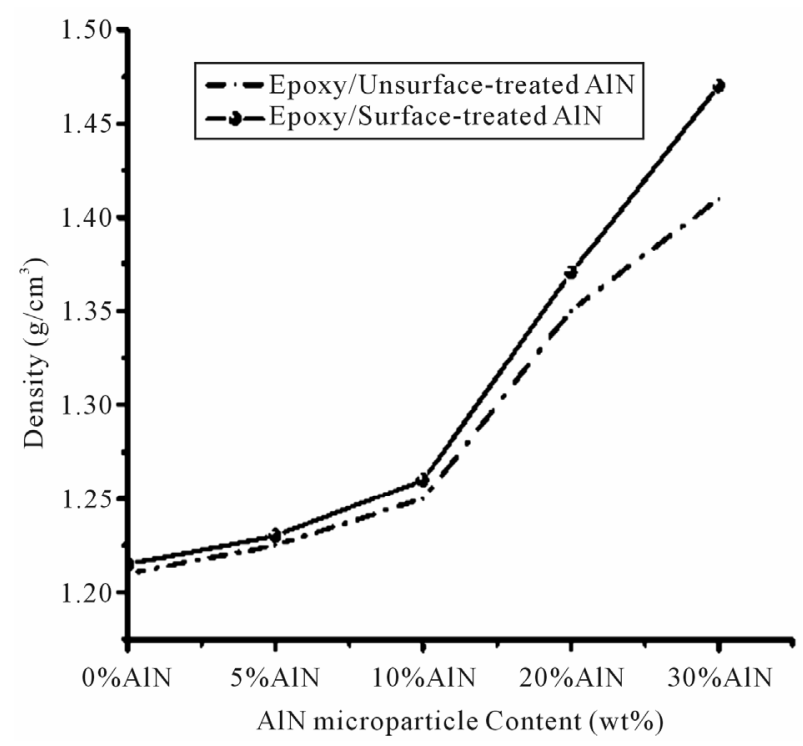

Figure 3. Density of virgin epoxy and epoxy/surface treated and unsurface-treated AIN composites.

Further, the density in the epoxy/AlN nanocomposites is depicted in the Table 2.

\subsection{Thermal Conductivity}

The variation of thermal conductivity of epoxy/AlN composites and nanocomposites is represented in Figures 4 and 5 and Table 3. Virgin epoxy shows a thermal conductivity value of $0.142 \mathrm{~W} / \mathrm{mK}$ at $25^{\circ} \mathrm{C}$ which subsequently increases to $0.167 \& 0.186 \mathrm{~W} / \mathrm{mK}$ with the increase in temperature up to $50^{\circ} \mathrm{C} \& 75^{\circ} \mathrm{C}$ respectively.

The incorporation of micro as well as nano AlN to the tune of 20 to $30 \mathrm{wt} \%$, results in an increase in the thermal
Table 2. Density of epoxy/unsurface-treated and surfacetreated AIN nanocomposites.

\begin{tabular}{ccc}
\hline \multirow{2}{*}{ Specimen Composition } & \multicolumn{2}{c}{ Density $\left(\mathbf{g} / \mathbf{c m}^{3}\right)$} \\
\cline { 2 - 3 } & $\begin{array}{c}\text { Epoxy/ } \\
\text { Unsurface-Treated } \\
\text { AlN }\end{array}$ & $\begin{array}{c}\text { Epoxy/ } \\
\text { Surface-Treated } \\
\text { AlN }\end{array}$ \\
\hline Epoxy + Hardener + 5\% nAlN & 1.231 & 1.242 \\
Epoxy + Hardener + 20\% nAlN & 1.362 & 1.382 \\
\hline
\end{tabular}

conductivity in the virgin matrix. At low loading of unsurface-treated AlN micro and nanoparticles of $5 \mathrm{wt} \%$, no significant increase in thermal conductivity of the matrix polymer was observed at $25^{\circ} \mathrm{C}$ and $50^{\circ} \mathrm{C}$. However, the conductivity increased with the increase in the loading levels of AlN from 10 to $20 \mathrm{wt} \%$, as there is a consistent increase in the conductivity values of the epoxy/AlN micro composites.

Further, surface treated epoxy/AIN micro composite revealed higher conductivity values as compared with the untreated samples. This behavior is probably due to the fact that surface modification minimizes the defects in the lattice structure of AlN, thus contributing to reduction in interfacial phonon scattering and decreasing the interface heat resistance while enhancing the conductivity. The epoxy/AlN micro composite at $20 \%$ AlN loading exhibits an optimum conductivity values, hence this composition has been retained for nanocomposite samples. A comparison with minimal loading of $5 \mathrm{wt} \%$ in epoxy/AlN has also been made with micro composite. It is evident that at low loading levels of nAlN also, there was no appreciable increase in the conductivity of the matrix. However, at $30 \mathrm{wt} \%$ of nAlN (both surface treated \& untreated), there was a significant increase in the conductivity of epoxy matrix when compared with micro composite. This is probably due to the presence of nanoscale platelets which creates an efficient pathway for conduction. The epoxy/surface-treated nAIN, revealed higher conductivity value, thus confirming efficient interfacial adhesion between the filler \& the matrix due to reduction in agglomeration of AlN nanoparticles with silane treatment. At higher loading of $30 \mathrm{wt} \%$ of AlN in the microcomposite, there was a decrease in thermal conductivity, which is possibly due to agglomeration of AlN particles that results in the formation of micro cracks at the interface in the composite [21]. In all the cases, conductivity increases with the increase in temperature which shows that there is an increase in interfacial adhesion at higher temperature.

\subsubsection{Relationship between Thermal Conductivity of Epoxy Composites and AIN Fractions}

It has been claimed that in case of nanofluids in which a small quantity of small particles (some nanometers in 


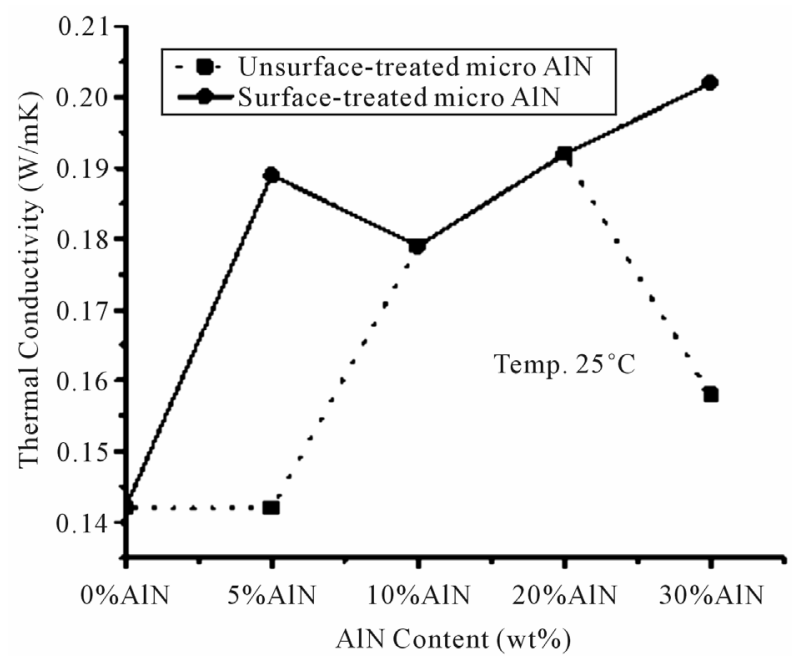

(a)

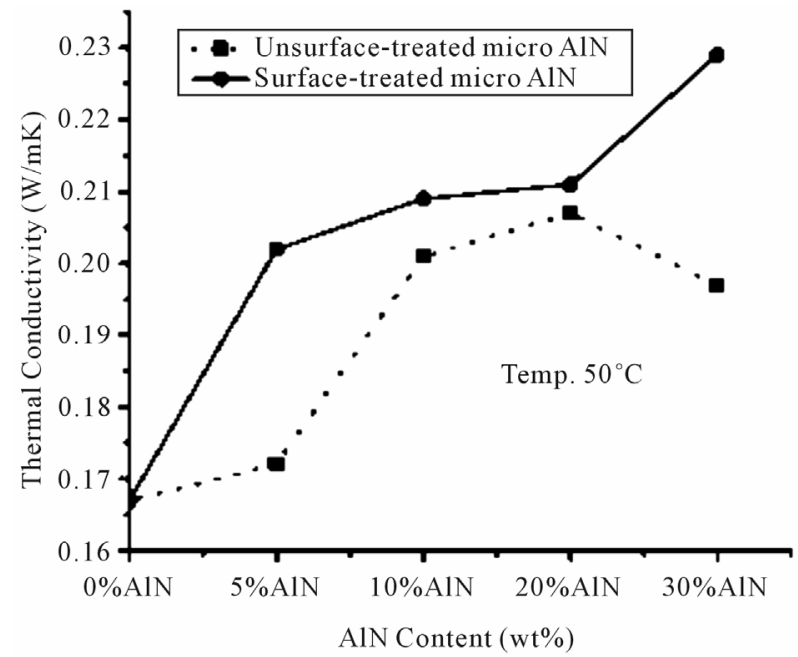

(b)

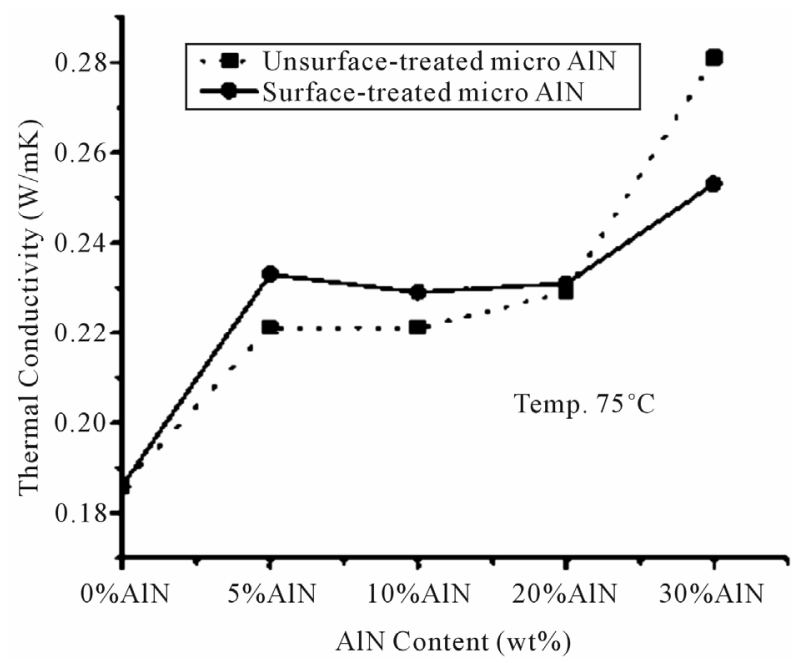

(c)

Figure 4. Thermal conductivity of epoxy/unsurface-treated AIN and epoxy/surface-treated AIN microcomposites at different temperatures: (a) $25^{\circ} \mathrm{C}$; (b) $50^{\circ} \mathrm{C}$; and (c) $75^{\circ} \mathrm{C}$.

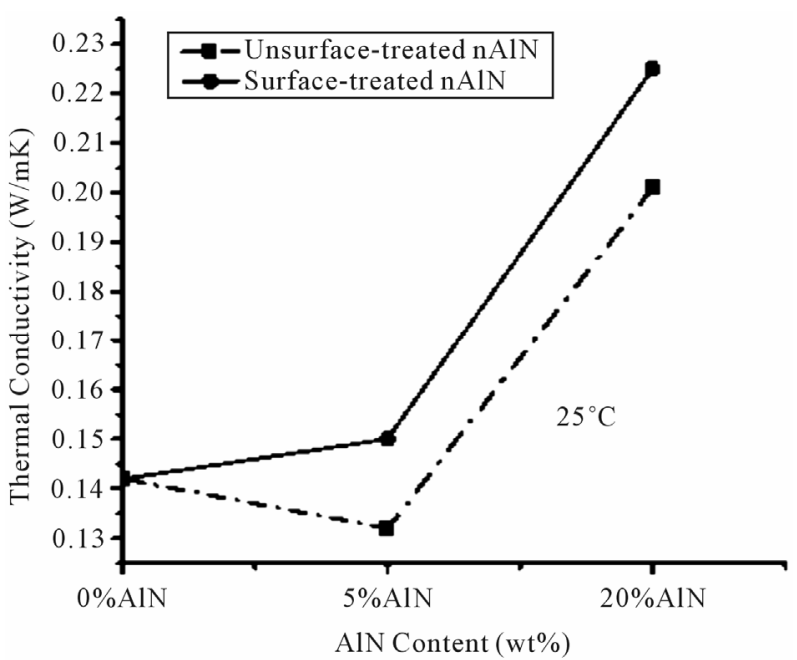

(a)

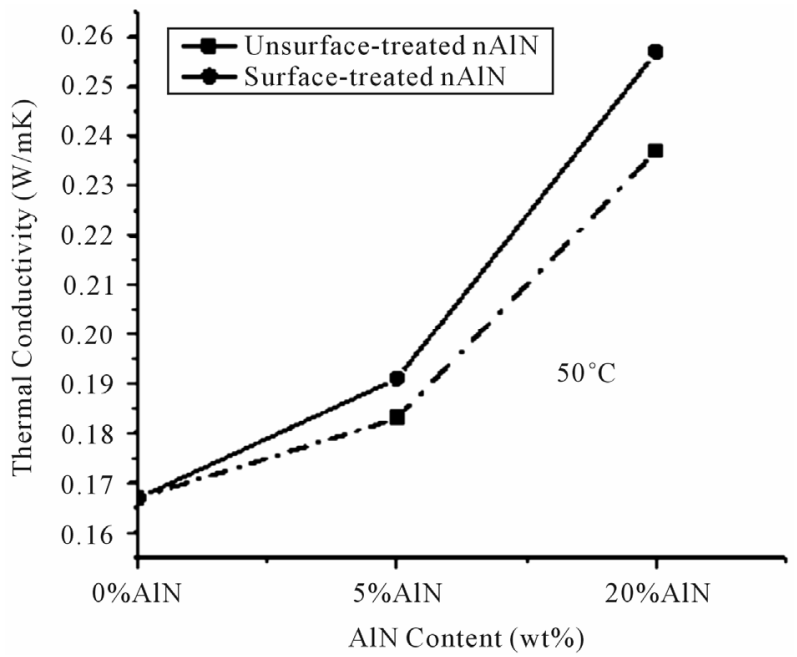

(b)

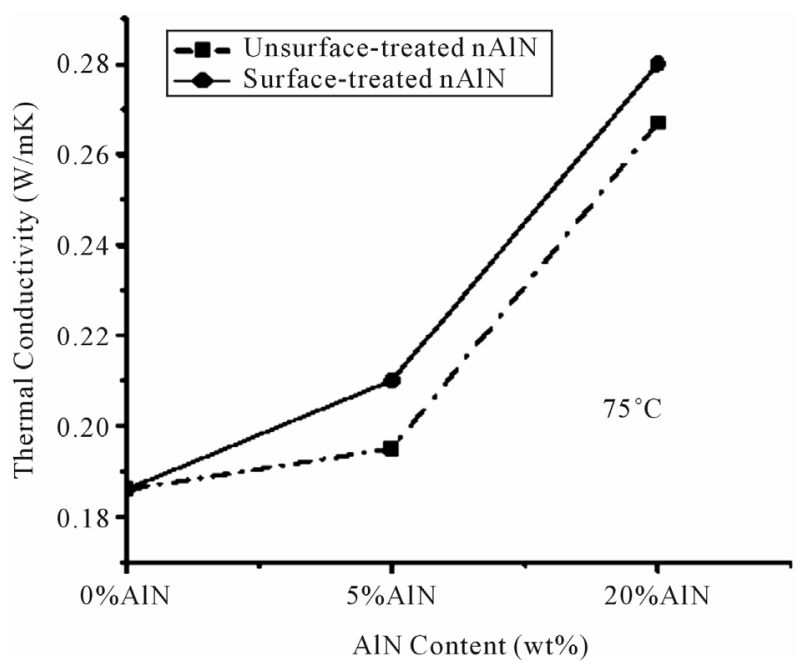

(c)

Figure 5. Thermal conductivity of epoxy/unsurface-treated AIN and epoxy/surface-treated AIN nanocomposites at different temperatures: (a) $25^{\circ} \mathrm{C}$; (b) $50^{\circ} \mathrm{C}$; and (c) $75^{\circ} \mathrm{C}$. 
Table 3. Variation of thermal conductivity of epoxy/AIN composites and nanocomposites.

\begin{tabular}{|c|c|c|c|c|c|c|}
\hline \multirow{4}{*}{ Specimen Composition } & \multicolumn{6}{|c|}{ Thermal Conductivity (W/mK) } \\
\hline & \multicolumn{3}{|c|}{ Unsurface-Treated AIN } & \multicolumn{3}{|c|}{ Surface-Treated AIN } \\
\hline & \multicolumn{6}{|c|}{ Temperature } \\
\hline & $25^{\circ} \mathrm{C}$ & $50^{\circ} \mathrm{C}$ & $75^{\circ} \mathrm{C}$ & $25^{\circ} \mathrm{C}$ & $50^{\circ} \mathrm{C}$ & $75^{\circ} \mathrm{C}$ \\
\hline Epoxy + Hardener $+0 \%$ micro AlN & 0.142 & 0.167 & 0.186 & 0.142 & 0.167 & 0.186 \\
\hline Epoxy + Hardener $+5 \%$ micro AlN & 0.142 & 0.172 & 0.221 & 0.189 & 0.202 & 0.233 \\
\hline Epoxy + Hardener $+10 \%$ micro AlN & 0.179 & 0.201 & 0.221 & 0.179 & 0.209 & 0.229 \\
\hline Epoxy + Hardener $+20 \%$ micro AlN & 0.192 & 0.207 & 0.229 & 0.192 & 0.211 & 0.231 \\
\hline Epoxy + Hardener $+30 \%$ micro AlN & 0.158 & 0.197 & 0.281 & 0.202 & 0.229 & 0.253 \\
\hline Epoxy + Hardener $+5 \%$ nAlN & 0.132 & 0.183 & 0.195 & 0.150 & 0.191 & 0.210 \\
\hline Epoxy + Hardener $+20 \%$ nAlN & 0.201 & 0.237 & 0.267 & 0.225 & 0.257 & 0.280 \\
\hline
\end{tabular}

size) present in a fluid matrix, gives very high thermal conductivity. As thermal conductivity, $k$, is the property of a material's ability to conduct heat as it appears primarily in Fourier's Law for heat conduction, the conductivity of AlN is much greater than that of epoxy. The addition of alluminium nitride filler to the epovy matrix thereby results in increase in thermal conductivity of the composite as in Figures $\mathbf{4}$ and 5. The thermal conductivity increases with increase in AlN fractions.

\subsubsection{Use of Kanari Model for Prediction of Thermal Properties of Polymer Composites}

Kanari model is a revised Bruggeman's equation and presents a relationship between the thermal conductivity of composites and the volume fractions of filler which is a function of the shape of filler:

$$
1-V_{f}=\frac{k_{c}-k_{f}}{k_{m}-k_{f}}\left(\frac{k_{m}}{k_{c}}\right)^{1 /(1+x)}
$$

where $V_{f}$ is the volume fraction of filler; $k_{m}$ is the thermal conductivity of matrix; $k_{f}$ is the thermal conductivity of AlN as filler; $k_{c}$ is the thermal conductivity of composite; $k_{m}$ is the thermal conductivity of matrix and $x$ is constant, determined by sphericity of the filler. For alluminium nitride, $x$ is substituted as 2.5 in Equation (1). The resullting model is given in Figure 6. Figure 6 clearly depicts the non-compliance of the experimental data curve with Kanari model. It is clear that each curve shows a small change in slope such as the thermal conductivity increases gradually with an increase in volume fraction of inorganic filler.

\subsection{Dielectric Break Down Strength}

Figures 7 and 8 depicts the dielectric break down strength of virgin epoxy and epoxy/AlN treated and un-

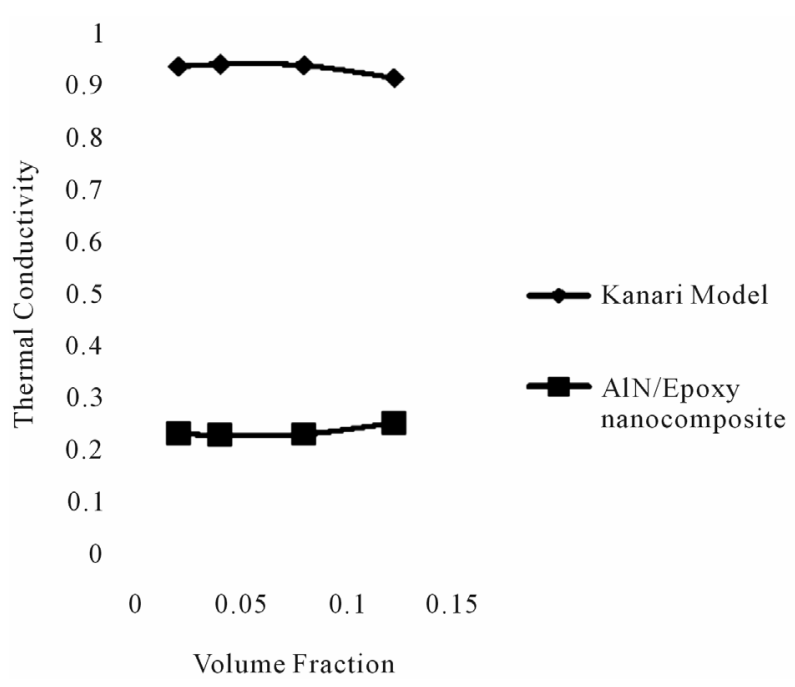

Figure 6. Thermal conductivity of the composite as a function of volume fraction of AIN.

treated micro composite at variable AlN loading. It is evident that break down strength of virgin epoxy increases marginally with the increase in AlN loading form 0 to $20(\mathrm{wt} \%)$. At filler concentration of $<10 \mathrm{wt} \%$, the break down strength of the untreated composite remains almost the same as the virgin matrix polymer.

This behavior is probably because at lower concentration of filler, the number of AlN particles is less, the interparticle distances are more and the volume fraction of the loose polymer layer is large under the condition of high electric stress, a large fraction of those polymer layer allow the transfer of charge carrier between the electrodes thereby loading to lower or marginally same breakdown voltage as the epoxy. However at higher filler content beyond $10 \mathrm{wt} \%$. i.e. at $20 \mathrm{wt} \%$ of AlN loading, the number of particles in the composite is larger with the inter particle distance being smaller, hence the volume 


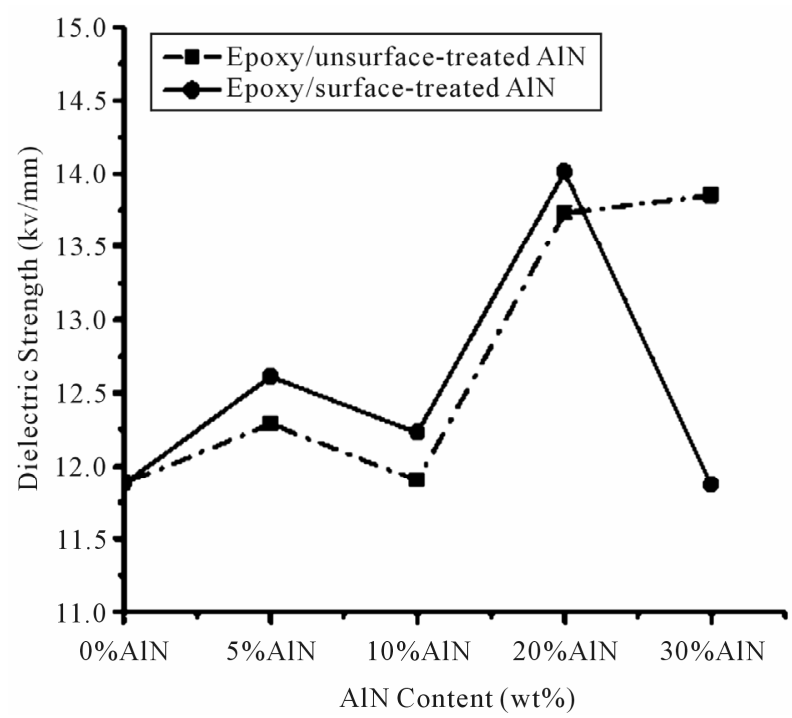

Figure 7. Dielectric break down strength of virgin epoxy and epoxy/AIN surface-treated and untreated micro composite at variable AIN loading.

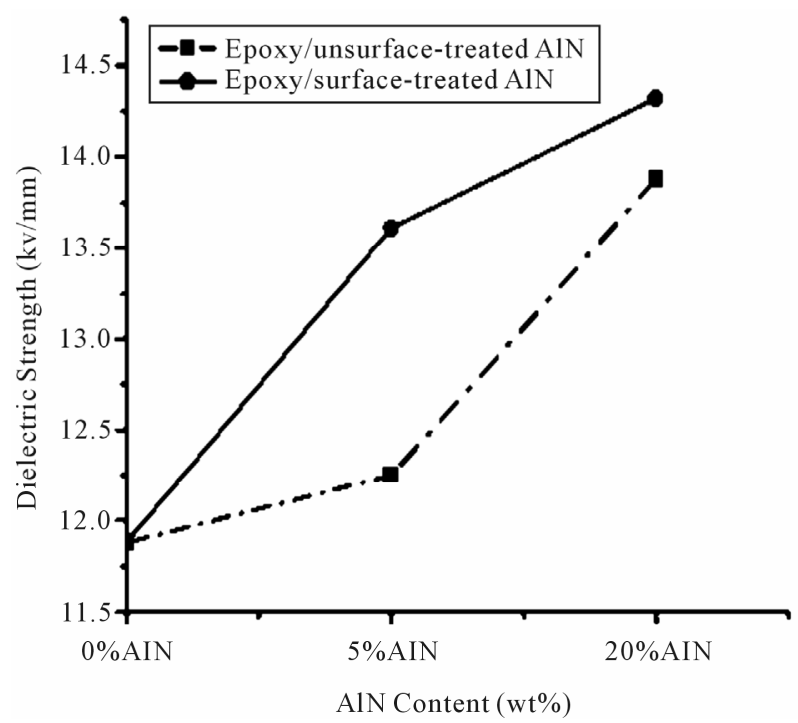

Figure 8. Dielectric break down strength of virgin epoxy and epoxy/surface-treated and untreated AIN nano composite at variable AIN loading.

fraction of loose polymer layer reduces and the particles themselves act as barriers to flow of current between the electrodes. These factors contribute to hindrance in the flow of current in the composite, resulting in higher breakdown voltage. Similar facts may also be applicable for epoxy/AlN nanocomposite at 5 and $20 \mathrm{wt} \%$ of nAlN as described in Table 4.

Further, all the surface treated epoxy/AlN micro and nanocomposite exhibited higher breakdown strength which reveals effective compatibility of the surfacetreated AlN with the matrix beyond $20 \mathrm{wt} \%$ of AlN, there was a reduction in the breakdown strength thus
Table 4. Dielectric strength of epoxy/(surface-treated and untreated) AIN nanocomposites.

\begin{tabular}{|c|c|c|}
\hline \multirow{3}{*}{ Specimen Composition } & \multicolumn{2}{|c|}{$\begin{array}{l}\text { Dielectric Break Down Strength } \\
\text { (KV/mm) }\end{array}$} \\
\hline & $\begin{array}{c}\text { Epoxy/ } \\
\text { Unsurface-Treated }\end{array}$ & $\begin{array}{c}\text { Epoxy/ } \\
\text { Surface-Treated }\end{array}$ \\
\hline & AIN & $\mathbf{A l N}$ \\
\hline Epoxy + Hardener $+5 \%$ nAlN & 12.25 & 13.61 \\
\hline Epoxy + Hardener $+20 \%$ nAlN & 13.88 & 14.32 \\
\hline
\end{tabular}

revealing agglomeration of the AlN micro particles thereby inducing voids and cracks within the matrix polymer [21].

\subsection{Volume Resistivity}

The volume resistivity of virgin epoxy, epoxy/AlN micro and nanocomposite at variable $\mathrm{wt} \%$ of $\mathrm{AlN}$ is represented in Figure 9 and Table 5. Virgin epoxy shows a resistivity value of $31.45 \times 10^{14} \Omega \cdot \mathrm{cm}$ which decreases with the incorporation of AlN micro as well as nanoparticles. It is evident that introduction of unsurface-treated AlN micro particles to the tune of 5 to $10 \mathrm{wt} \%$ substantially reduces the resistivity value to $0.26-0.43 \Omega \cdot \mathrm{cm}$, which subsequently increases with higher filler loading. This behavior indicates that at lower filler loading, the fraction of extended loose polymer layers is high which probably allows the existence of free ions and also their unhindered transport through the bulk of the material those results in an increase in the electrical conductivity through the volume of the material [22]. However, with the increase in AlN loading up to $20 \mathrm{wt} \%$, immobilized polymer chains act as ion traps which inhibit the ion mobility thereby resulting in decrease in the overall conductivity values. Further, the epoxy/surface-treated AIN microcomposite exhibited higher resistivity values as compared with the untreated samples at similar wt $\%$ of AlN loading. This further reveals improved interfacial adhesion at the interface which hinders the flow of electrons, thus giving a less conductivity.

The volume resistivity of epoxy/AIN nanocomposite is also displayed in the Table 5. As observed, the nanofilled system (both surface-treated and untreated) display lesser conductivity (higher resistivity) as compared with the microcomposites at same loading. This decrease in conductivity is primarily due to hindered conductive path in the nanocomposite by the highly concentrated AlN networks.

\subsection{Dynamic Mechanical Analysis (DMA)}

Figure 10 shows the storage modulus attained, as a function of the temperature for the various epoxy systems. The figure clearly shows that the storage modulus of neat 
Table 5. Volume resistivity of virgin epoxy, epoxy/AIN micro and nanocomposite at variable wt\% of AIN.

\begin{tabular}{lcc}
\hline \multirow{2}{*}{ Specimen Composition } & \multicolumn{2}{c}{ Volume Resistivity $(\mathbf{\Omega} \cdot \mathbf{c m})$} \\
\cline { 2 - 3 } & Epoxy/Unsurface-Treated AIN & Epoxy/Surface-Treated AIN \\
\hline Epoxy + Hardener + 0\% micro AlN & $31.45 \times 10^{14}$ & $31.45 \times 10^{14}$ \\
Epoxy + Hardener + 5\% micro AlN & $0.260 \times 10^{14}$ & $2.92 \times 10^{14}$ \\
Epoxy + Hardener + 10\% micro AlN & $0.432 \times 10^{14}$ & $7.06 \times 10^{14}$ \\
Epoxy + Hardener + 20\% micro AlN & $5.14 \times 10^{14}$ & $7.16 \times 10^{14}$ \\
Epoxy + Hardener + 30\% micro AlN & $4.28 \times 10^{14}$ & $4.33 \times 10^{14}$ \\
Epoxy + Hardener + 5\% nAlN & $6.84 \times 10^{14}$ & $7.31 \times 10^{14}$ \\
Epoxy + Hardener + 20\% nAlN & $8.84 \times 10^{14}$ & $10.5 \times 10^{14}$ \\
\hline
\end{tabular}

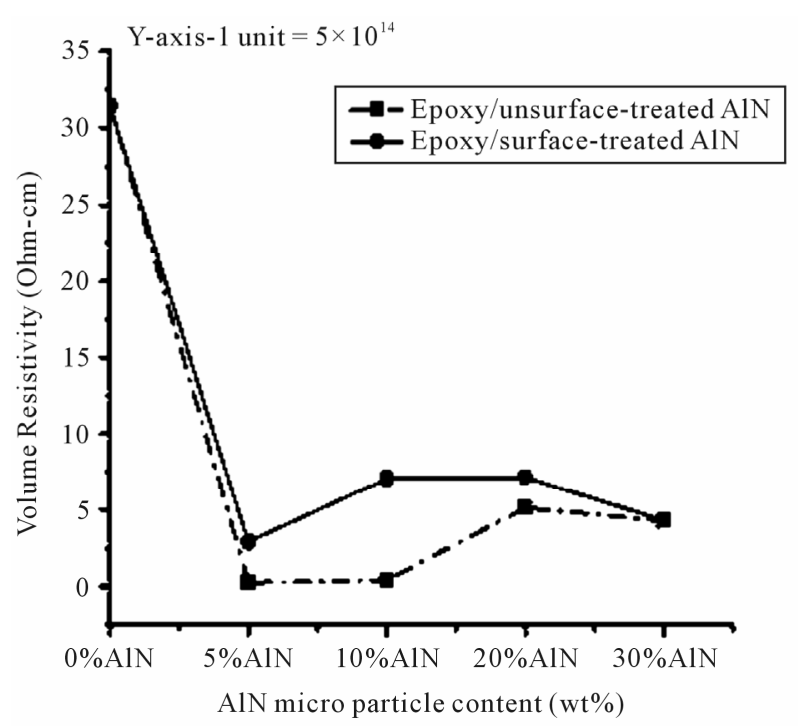

(a)

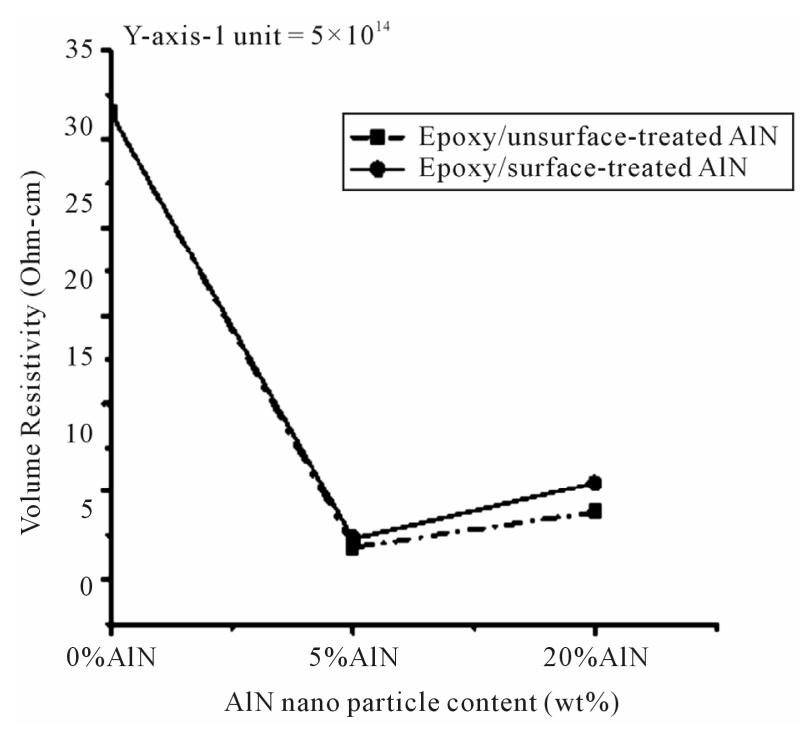

(b)

Figure 9. Volume resistivity of virgin epoxy and (a) epoxy/ AIN micro; (b) epoxy/AIN nanocomposite.

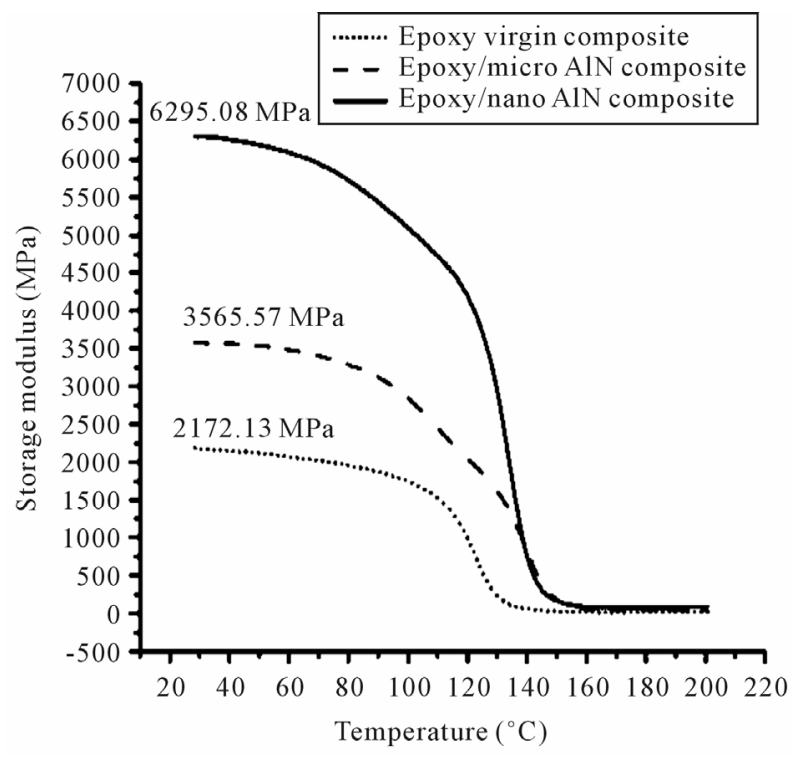

Figure 10. Storage modulus versus temperature of Virgin, micro and nano composite.

epoxy, epoxy/micro AlN and epoxy/nAlN composites are 2172.13 $\mathrm{MPa}$, 3565.57 $\mathrm{MPa}$ and 6295.08 $\mathrm{MPa}$ respectively. In the glassy state the incorporation of micro aluminium nitride (AIN) yielded a $64.15 \%$ increase in storage modulus as compared to virgin epoxy, which further increased by $76.55 \%$ due to addition of nano AlN particles. In a rubbery state the modulus of virgin epoxy is equal to the micro \& nano composite. This behavior can be explained in terms of possible physical interaction between the micro/nano AlN with virgin epoxy matrix through their enormous interfacial area. The modification with nano AlN particle implies a greater value of storage modulus (6295.08 $\mathrm{MPa}$ ) which is due to the adhesion of nano filler into the epoxy matrix resulting in the stiffness and sufficient energy storage in the material.

It can be definitely considered that the homogeneous dispersion and the excellent interfacial coupling force in a mixture state of the micro and nano particles limits the 
mobility of the epoxy molecules and enhance the storage modulus before Tg $[23,24]$.

Loss modulus curves of the neat epoxy, epoxy/micro AlN and epoxy/nAlN composites are shown in Figure 11. In this the $\mathrm{Tg}$ value of micro composite is $137.90^{\circ} \mathrm{C}$ where as the $\mathrm{Tg}$ value of virgin and nano composite are $122.97^{\circ} \mathrm{C}$ and $134.26^{\circ} \mathrm{C}$ respectively.

The $\mathrm{Tg}$ of composite increased by 15 degree Celsius from $122.97^{\circ} \mathrm{C}$ (neat epoxy) to $137.90^{\circ} \mathrm{C}$ (micro epoxy composite). The shift of $\mathrm{Tg}$ for particulate-filled micro composite reaches a higher temperature due to immobility of polymer molecules caused by local adsorption onto the particles. The average interparticle distance decreases with the incorporation of micro particles rather than nanoparticles, resulting in the decrease in volume fraction of loosely bound polymer. The peak height of the tan delta and the area under tan delta curve of composite and nano composite decreased substantially with the loading of AlN particles. The peak value (131.27) of neat epoxy is reduced to epoxy/micro AlN composite. Similarly the peak value of tan delta is reduced considerably in epoxy/ AlN nano composite as compared to the neat epoxy. This is probably due to the effect of increase in crystallinity, the reduced fraction of epoxy composite and nano composite and homogeneous dispersion of AIN nanoparticles in epoxy matrix, results in a strong interaction on physical bonding between AlN particles micro, nano and virgin epoxy matrix. It indicates that micro composite gave excellent thermal property than virgin epoxy and nano composite [26].

Loss modulus (E") before and after Tg keep constant with temperature but storage modulus ( $\left.E^{\prime}\right)$ before and after $\mathrm{Tg}$ varies with temperature this decrease in storage modulus in the glassy and rubbery state may be due to different coefficient of thermal expansion (CTE) of the matrix and filler, inducing relaxation in the polymeric phase and considering that the decrease of storage modulus $\mathrm{E}^{\prime}$ with temperature in glassy and rubbery state is small [25]. The representation value $E^{\prime} \& E^{\prime \prime}$ in the glassy state are taken at about $28^{\circ} \mathrm{C}$ and the representation value of $\mathrm{E}^{\prime} \& \mathrm{E}^{\prime \prime}$ in the rubbery state are taken at about $200^{\circ} \mathrm{C}$.

Tan delta curves of the micro, nano and neat epoxy composite are shown in Figure 12. It is observed that converse value that is tan delta represents a temperature difference of $18.01^{\circ} \mathrm{C}$ for micro composite and temperature difference of $11.39^{\circ} \mathrm{C}$ for nano composite in comparison to virgin epoxy composite.

\subsection{Morphological Analysis: Scanning Electron Microscopy (SEM)}

The micro structure of $20 \mathrm{wt} \%$ AlN loaded epoxy composite and nanocomposites (both surface-treated \& untreated) are shown in Figures 13 and 14. In case of both

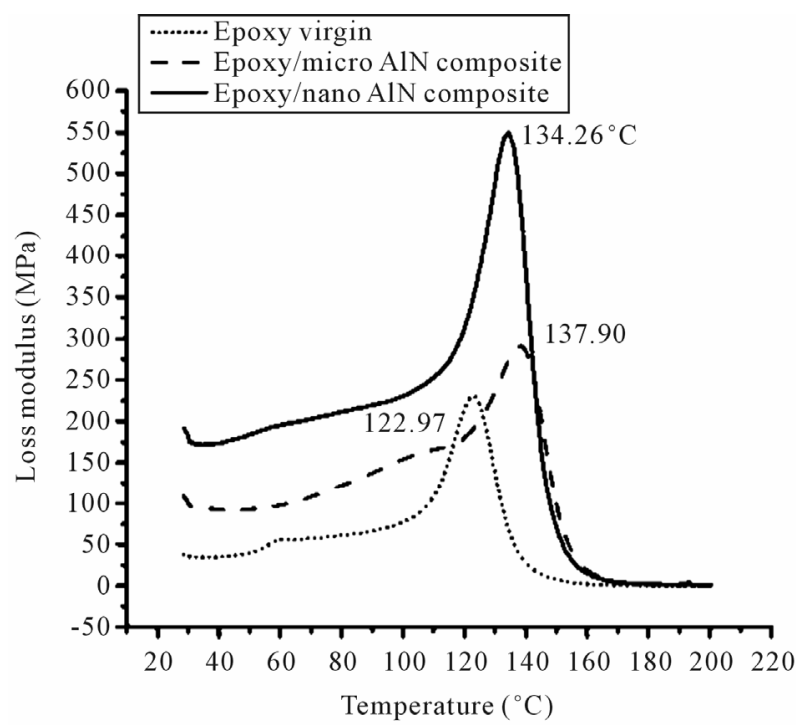

Figure 11. Loss modulus versus temperature of Virgin, micro and nanocomposite.

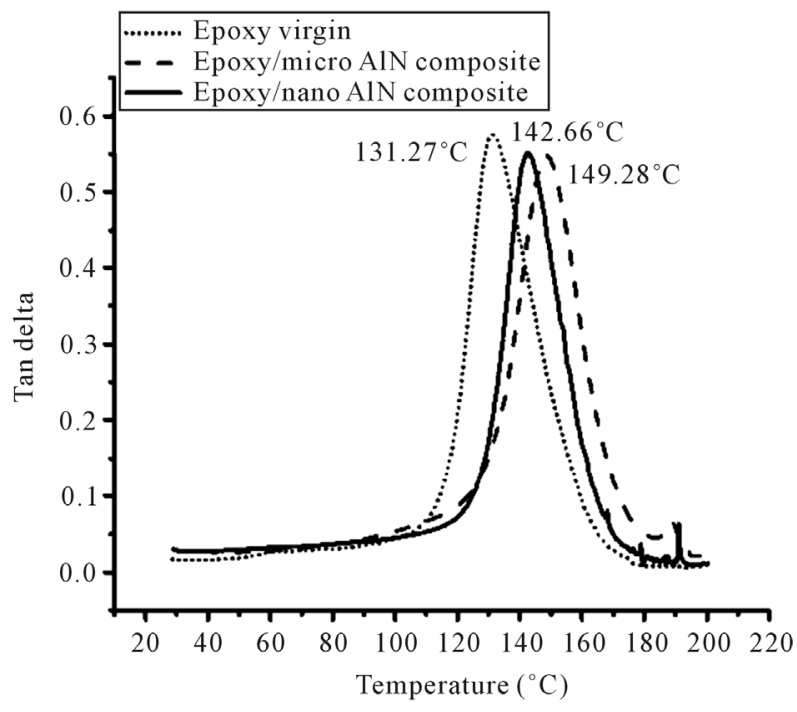

Figure 12. Tan delta versus temperature of Virgin, micro and nanocomposite.

surface-treated epoxy/AlN nano composite and micro composites, silane coupling agent on the AIN surface is found playing a significant role like an adhesion promoter between filler particles and epoxy matrix. The homogeneous dispersion of micro AIN in the epoxy matrix as compared to the AIN nanoparticles is clearly observed in Figures 13(b), 13(c), 14(a) and 14(b). Figure 13(a) indicates the SEM micrograph of virgin epoxy matrix showing the fracture surface, which is further modified with the addition of AlN particles showing the enhancement of interaction within the layers of neat epoxy matrix and the silane treated AIN micro composite and nano composite.

Both the case of unsurface-treated epoxy/AlN micro 


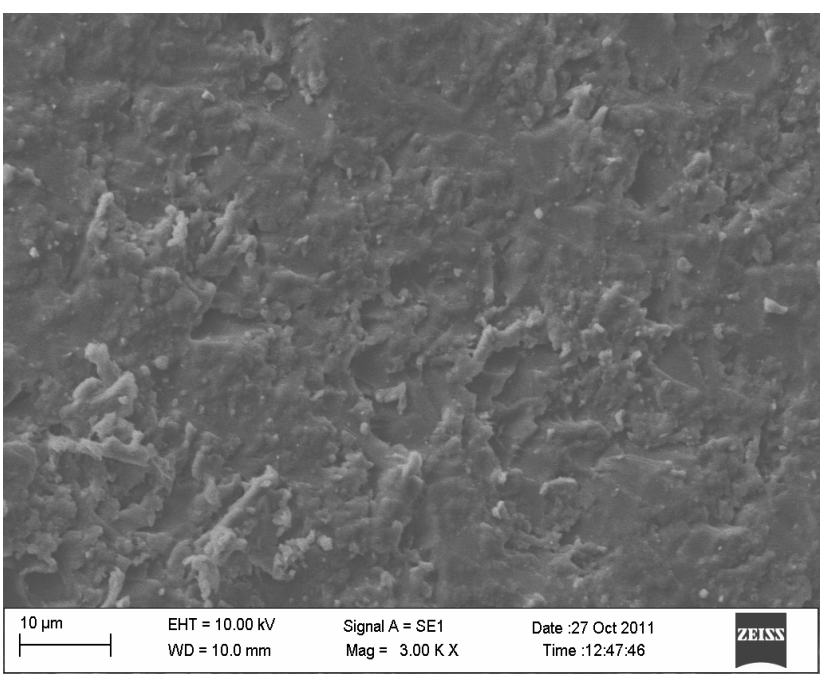

(a)

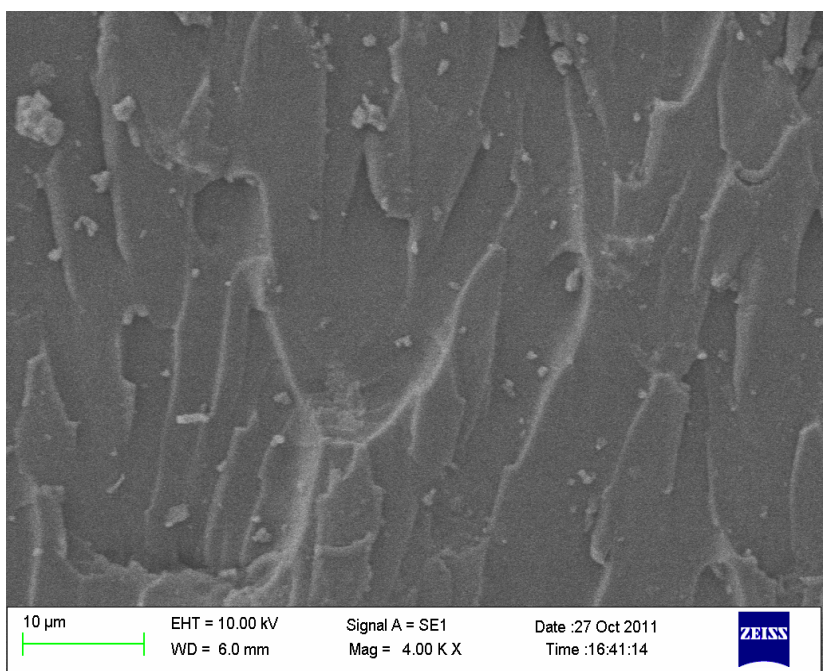

(c)

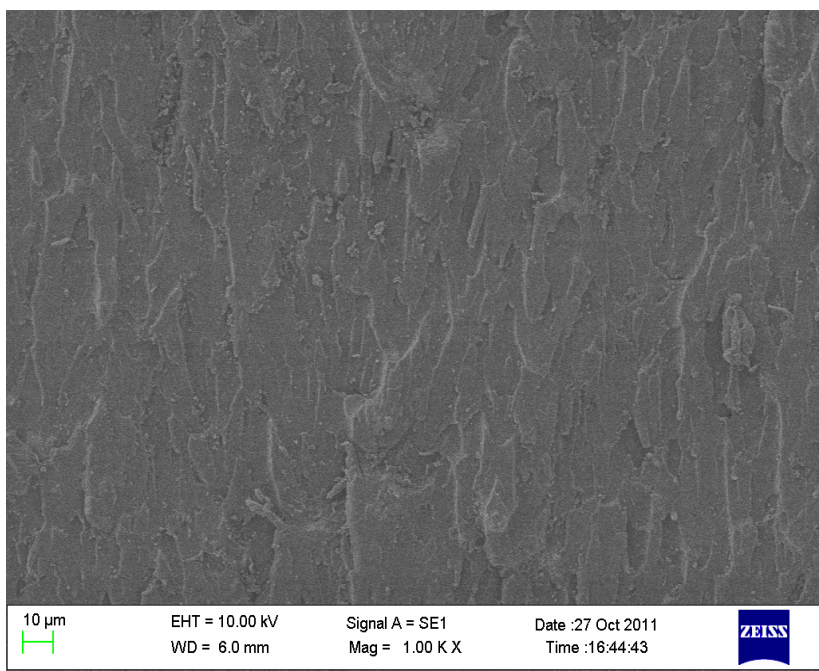

(b)

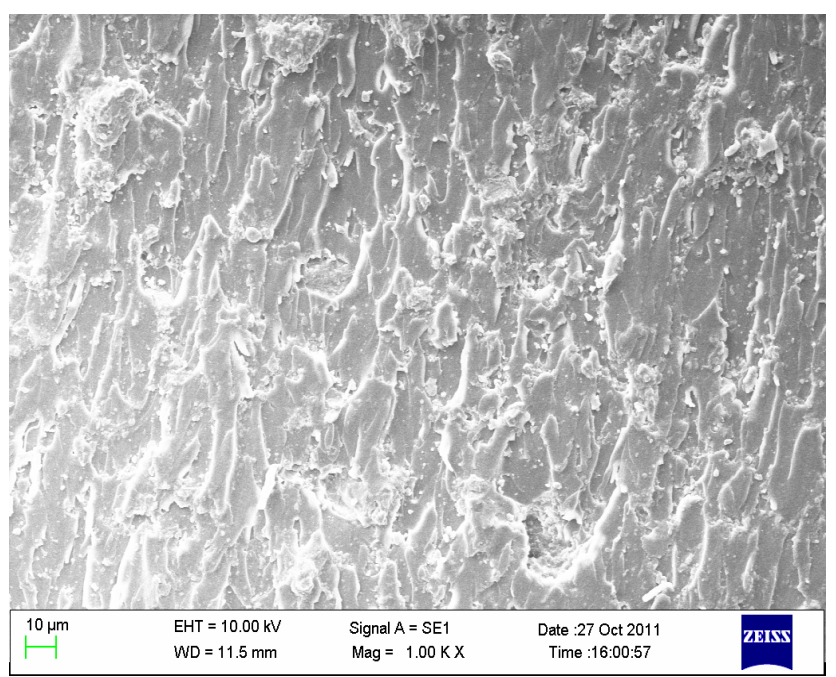

(d)

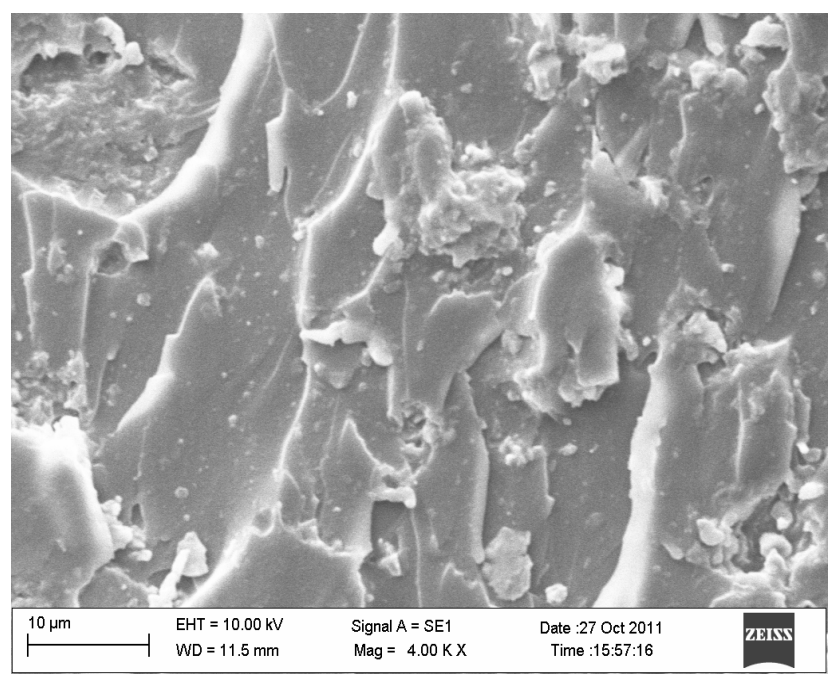

(e)

Figure 13. SEM images of: (a) neat epoxy, (b), (c) epoxy with $20 \%$ surface-treated micro AlN and (d), (e) epoxy with $20 \%$ unsurface-treated micro AIN. 


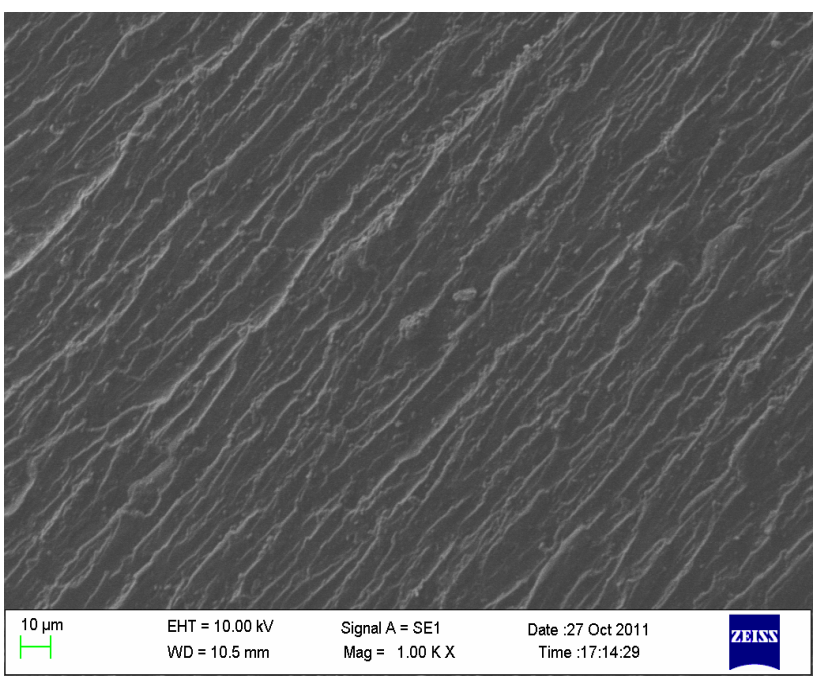

(a)

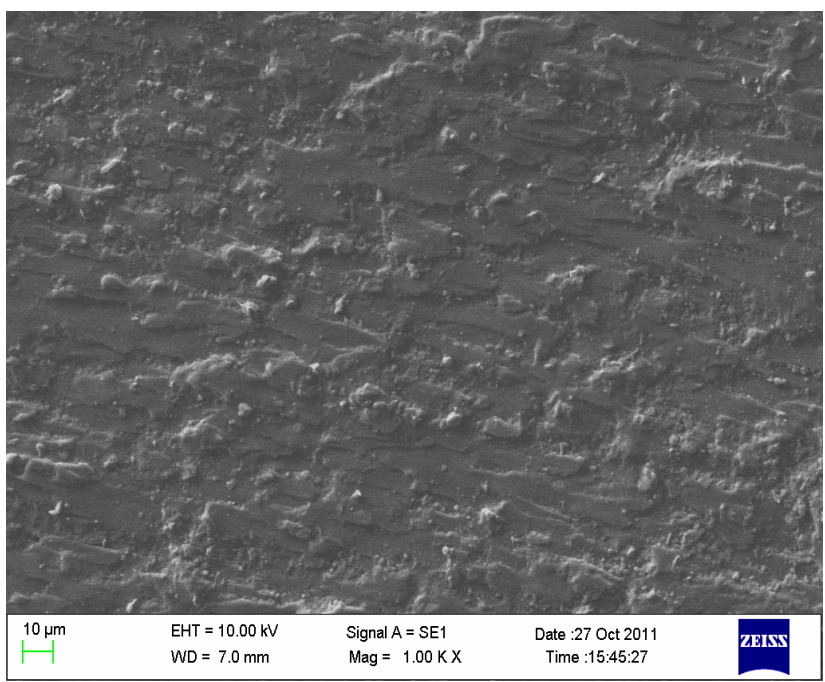

(c)

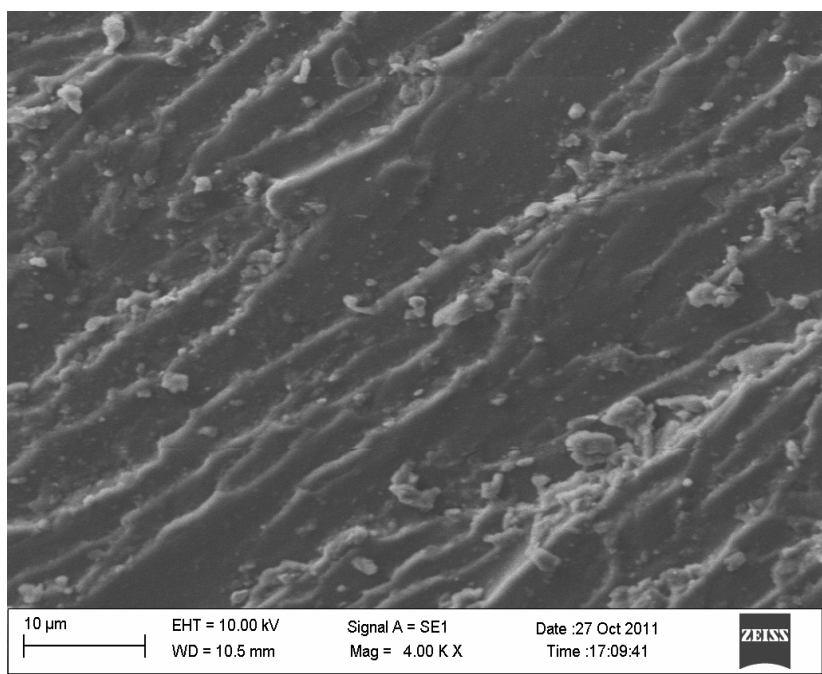

(b)

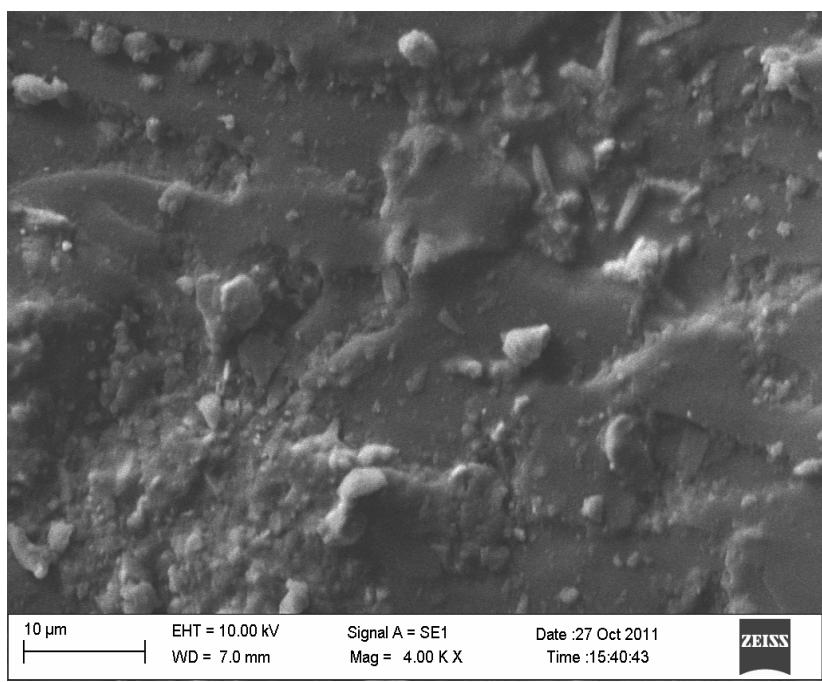

(d)

Figure 14. SEM images of: (a), (b) epoxy with $20 \%$ surface-treated nano AIN and (c), (d) epoxy with $20 \%$ unsurface-treated nano AIN.

composites and nano composites is illus trated in Figures 13(d), 13(e), 14(c) and 14(d), wherein the AlN particles are agglomerated on the epoxy surface. These particles are not properly embedded in to the matrix, resulting in uneven flake like surface. The compatibility of the AlN nanocomposite as compared with virgin epoxy matrix is meager as compared to the microcomposite which is clearly shown in the Figures 13(a)-(c), 14(a) and 14(b) respectively.

\section{Conclusions}

The following conclusions have been drawn from the present study towards effective behavior of surface modification of nano and micro particles on the dynamic mechanical strength, glass transition temperature, electrical property and thermal conductivity and morphology of epoxy/AIN composites.

- The silane incorporation in composite yielded improved electrical and thermal property when compared with those loaded with unsurface-treated nanoparticles.

- The incorporation of silane in epoxy/AlN composite and nanocomposite result in a better compatibility and cross linking reveals to an increase in dielectric break down strength, thermal conductivity and glass transition temperature when compared with the unsurfacetreated epoxy/AlN composite and nanocomposites.

- The epoxy/AIN nanocomposites showed lower value for volume resistivity when compared to virgin epoxy at lower filler loading, due to better interfacial adhesion, again. The conductivity falls to alower value.

- At $20 \mathrm{wt} \%$ of nAIN both (surface-treated and un- 
treated), there was a significant increase in the conductivity of epoxy matrix when compared with composite. This is due to the existence of nanoscale platelets which forms the channel for conduction.

- A significant change was noted in storage modulus of the epoxy/AIN microcomposite up to the vicinity of the Tg of the epoxy. The Tg of the epoxy was also affected moderately by the addition of AIN nano particles. Since stiffness and strength appear to increase with the incorporation of AIN micro and nano particles.

- The result indicates the AIN nano and micro particles dispersion is adequate which may induce some structure change in the epoxy matrix reflects on its viscoelastic properties.

\section{REFERENCES}

[1] W. Kim, J. Bae and I. Choi, "Thermally Conductive EMC (Epoxy Molding Compound) for Microelectronic Encapsulation," Polymer Engineering and Science, Vol. 39, No. 4, 1999, pp. 756-766. doi:10.1002/pen.11464

[2] M. Ohashi, S. Kawakami, Y. Yokogawa and G. C. Lai, "Spherical Aluminum Nitride Fillers for Heat-Conducting Plastic Packages," Journal of the American Ceramic Society, Vol. 88, No. 9, 2005, pp. 2615-2618. doi:10.1111/j.1551-2916.2005.00456.x

[3] P. Procter and J. Solc, "Improved Thermal Conductivity in Microelectronic Encapsulants," IEEE Transactions on Components Hybrids and Manufacturing Technology, Vol. 14, No. 4, 1991, pp. 708-713. doi: $10.1109 / 33.105121$

[4] P. Kuhnlein, S. Ino and T. Shiobara, "Thermal Conductivity of Molding Compounds for Plastic Packaging," IEEE Transactions on Components Packaging and Manufacturing Technologies Part A, Vol. 17, No. 4, 1994, pp. 527-532.

[5] C. P. Wong and R. S. Bollampally, "Thermal Conductivity, Elastic Modulus, and Coefficient of Thermal Expansion of Polymer Composites Filled with Ceramic Particles for Electronic Packaging," Journal of Applied Polymer Science, Vol. 74, No. 14, 1999, pp. 3396-3403. doi:10.1002/(SICI)1097-4628(19991227)74:14<3396::AI D-APP13>3.0.CO;2-3

[6] L. C. Sim, S. R. Ramanan, H. Ismail, K. N. Seetharamu and T. J. Goh, "Thermal Characterization of $\mathrm{Al}_{2} \mathrm{O}_{3}$ and $\mathrm{ZnO}$ Reinforced Silicone Rubber as Thermal Pads for Heat Dissipation Purposes," Journal of Thermochimica Acta, Vol. 430, No. 1-2, 2005, pp. 155-165. doi:10.1016/j.tca.2004.12.024

[7] G. W. Lee, M. Park, J. Kim, J. I. Lee and H. G. Yoon, "Enhanced Thermal Conductivity of Polymer Composites Filled with Hybrid Filler," Composite A: Applied Science \& Manufacturing, Vol. 37, No. 5, 2006, pp. 727-734. doi:10.1016/j.compositesa.2005.07.006

[8] K. C. Yung, B. L. Zhu1, J. Wu, T. M. Yue and C. S. Xie, "Effect of AIN Content on the Performance of Brominated Epoxy Resin for Printed Circuit Board Substrate,"
Journal of Polymer Science Part B: Polymer Physics, Vol. 45, No. 13, 2007, pp. 1662-1674.

[9] I. Hatsuo and S. Rimdusit, "Very High Thermal Conductivity Obtained by Boron Nitride-Filled polybenzoxazine," Thermochim Acta, Vol. 320, No. 1-2, 1998, pp. 177 186.

[10] Y. Nagai and G. C. Lai, "Thermal Conductivity of Epoxy Resin Filled with Particulate Aluminum Nitride Powder," Journal of the American Ceramic Society, Vol. 105, No. 1219, 1997, pp. 197-200. doi:10.2109/jcersj.105.197

[11] C. Hsieh and S. Chung, "High Thermal Conductivity Epoxy Molding Compound Filled with a Combustion Synthesized AIN Powder," Journal of Applied Polymer Science, Vol. 102, No. 5, 2006, pp. 4734-4740. doi:10.1002/app. 25000

[12] S. Xie, B. Zhu, J. Li, X. Wei and Z. Xu, "Preparation and Properties of Polyimide/Aluminum Nitride Composites," Polymer Testing, Vol. 23, No. 7, 2004, pp. 797-801. doi:10.1016/j.polymertesting.2004.03.005

[13] S. Z. Yu, P. Hing and X. Hu, "Thermal Conductivity of Polystyrene-Aluminum Nitride Composite," Composite A: Applied Science Manufacturing, Vol. 33, No. 2, 2002, pp. 289-292. doi:10.1016/S1359-835X(01)00107-5

[14] Y. S. Xu, D. D. L. Chung and C. Mroz, "Thermally Conducting Aluminum Nitride Polymer-Matrix Composites," Composite A: Applied Science Manufacturing, Vol. 32, No. 12, 2001, pp. 1749-1757. doi:10.1016/S1359-835X(01)00023-9

[15] Y. S. Xu and D. D. L. Chung, "Increasing the Thermal Conductivity of Boron Nitride and Aluminium Nitride Particle Epoxy Matrix Composites by Particle Surface Treatments," Compos Interfaces, Vol. 7, No. 4, 2000, pp. 243-256. doi:10.1163/156855400750244969

[16] J. J. Wang and X. S. Yi, "Effects of Interfacial Thermal Barrier Resistance and Particle Shape and Size on the Thermal Conductivity of AIN/PI Composites," Composite Science Technology, Vol. 64, No. 10-11, 2004, pp. 16231628. doi:10.1016/j.compscitech.2003.11.007

[17] J. W. Bae, W. Kim, S. H. Cho and S. H. Lee, "The Properties of AlN-Filled Epoxy Molding Compounds by the Effects of Filler Size Distribution," Journal of Material Science, Vol. 35, No. 23, 2000, pp. 5907-5913. doi:10.1023/A:1026741300020

[18] G. Pezzotti, I. Kamada and S. Miki, "Thermal Conductivity of AlN/Polystyrene Interpenetrating Networks," Journal of the European Ceramic Society, Vol. 20, No. 8, 2000, pp. 1197-1203. doi:10.1016/S0955-2219(99)00282-4

[19] S. Kume, I. Yamada, K. Watari, I. Harada and K. Mitsuishi, "High-Thermal-Conductivity AIN Filler for Polymer/Ceramics Composites," Journal of the American Ceramic Society, Vol. 92, Suppl. 1, 2009, pp. 153-156 doi:10.1111/j.1551-2916.2008.02650.x

[20] M. Ohashi, S. Kawakami, Y. Yokogawa and G. Lai, "Spherical Aluminum Nitride Fillers for Heat-Conducting Plastic Packages," Journal of the American Ceramic Society, Vol. 88, No. 9, 2005, pp. 2615-2618. doi:10.1111/j.1551-2916.2005.00456.x 
[21] W. Peng, X. Huang, J. Yu, P. Jiang and W. Liu, "Electrical and Thermophysical Properties of Epoxy/Aluminum Nitride Nanocomposites: Effect of Nanoparticle Surface Modification," Composites Part A: Applied Science and Manufacturing, Vol. 41, No. 9, 2010, pp. 1201-1209. doi:10.1016/j.compositesa.2010.05.002

[22] S. Singha and M. J. Thomas, "Dielectric Properties of Epoxy Nanocomposites," IEEE Transactions on Dielectrics and Electrical Insulation, Vol. 15, No. 1, 2008, pp. 1070-9878. doi:10.1109/T-DEI.2008.4446732

[23] L. Y. Lin, J. H. Lee, C. E. Hong, G. H. Yoo and S. G. Advani, "Preparation and Characterization of Layered Silicate/Glass Fiber/Epoxy Hybrid Nanocomposites via Vacuum-Assisted Resin Transfer Molding (VARTM),"
Composites Science and Technology, Vol. 66, No. 13, 2006, pp. 2116-2125. doi:10.1016/j.compscitech.2005.12.025

[24] W. Chia-Chung, C. Ying-Chung, Y. Cheng-Fu, S. CheanCheng and D. Chein-Chen, "The Dielectric Properties of Epoxy/AlN Composites," Journal of the European Ceramic Society, Vol. 27, 2007, pp. 3839-3842.

[25] M. Preghenella, A. Pegoretti and C. Migliaresi, "ThermoMechanical Characterization of Fumed Silica-Epoxy Nanocomposites," Polymer, Vol. 46, No. 26, 2005, pp. 12065 12072. doi:10.1016/j.polymer.2005.10.098

[26] C. H. Lee and J. J. Park, "The Properties of DSC and DMA for Epoxy Nano-and-Micro Mixture Composites," Transactions on Electrical and Electronic Materials, Vol. 11, No. 2, 2010, pp. 69-72. 